\title{
BOUNDED ANALYTIC FUNCTIONS AND THE LITTLE BLOCH SPACE ROHAN ATTELE
}

\author{
Department of Mathematics \\ University of North Carolina at Charlotte \\ Charlotte, NC 28223
}

(Received April 24, 1984 and in revised form May 29, 1989)

ABSTRACT. The radial limits of the weighted derivative of an bounded analytic function is considered.

KEY WORDS AND PHRASES. Bloch space, Bounded analytic functions.

1980 AMS SUBECT CLASSIFICATION OODES. Primary 30A, Secondary 30H05

\section{INTRODUCTION.}

Let $D$ denote the unit disc of the complex plane $C$ and let $H^{\infty}$ denote the space of bounded analytic functions on D. An analytic function on D is called a Bloch function if $\operatorname{Sup}_{z \in D}\left|f^{\prime}(z)\right|\left(1-|z|^{2}\right)<\infty$. The space $B$ of Bloch functions is $a$ Banach space with norm

$$
\|f\|_{\mathscr{B}}=|f(0)|+\sup _{z \in D}\left|f^{\prime}(z)\right|\left(1-|z|^{2}\right) .
$$

A Bloch function is in the little Bloch space ${ }_{0}^{\circ}$, if $f^{\prime}(z)\left(1-|z|^{2}\right) \rightarrow 0$ as $|z|$ $\rightarrow$ 1-. An immediate consequence of Schwartz lemma (see for example [2], Lemma 1.2) is that $H^{\infty} C \mathscr{B}$, however it is well known (see section 3 for an explicit example) that $H^{\infty} \notin{ }_{0}$. The main result of this paper is to show that, if $f \in H^{\infty}$ then $f^{\prime}\left(r e^{i \theta}\right)\left(1-r^{2}\right) \rightarrow 0$ for almost all $\theta$ as $r \rightarrow 1-$.

\section{APPROXIMATE IDENTITY.}

In this section we establish an approximate identity akin to the Poisson Kernel.

LEMMA 1. Let $0<\mathrm{r}<1, \mathrm{t} \in \mathbb{R}$ and 


$$
p(r, t)=\frac{\left(1-r^{2}\right)^{3}}{\left(1+r^{2}\right)} \frac{1}{\left(1-2 r \cos t+r^{2}\right)^{2}}
$$

Then $\frac{1}{2 \pi} \int_{-\pi}^{\pi} p(r, t) d t=1$

PROOF: Let $\mathrm{P}_{\mathrm{r}}(t)=\frac{1-\mathrm{r}^{2}}{1-2 r \cos t+\mathrm{r}^{2}}=1+2 \sum_{1}^{\infty} \mathrm{r}^{\mathrm{n}} \cos \mathrm{nt}$ be the Poisson kernel. As usual let $L^{2}$ be the Lebesgue 2-space on $[0,2 \pi]$. Then,

$$
\begin{aligned}
\frac{1}{2 \pi} \int_{-\pi}^{\pi} p(r, t) d t & =\frac{\left(1-r^{2}\right)}{\left(1+r^{2}\right)}\left\|p_{r}(t)\right\|_{L^{2}}^{2} \\
& =\frac{\left(1-r^{2}\right)}{\left(1+r^{2}\right)}\left\langle 1+2 \sum_{1}^{\infty} r^{n} \cos n t, 1+2 \sum_{1}^{\infty} r^{n} \cos n t\right\rangle \\
& =\frac{1-r^{2}}{1+r^{2}}\left(1+2 \sum_{1}^{\infty} r^{2 n}\right)=1 .
\end{aligned}
$$

LEMA 2. Let $\beta$ be a complex measure on $[-\pi, \pi]$ and suppose the derivative $\mathrm{D} \mu(\theta)$ exists for some point $\theta$. Then

$$
\frac{1}{2 \pi} \int_{-\pi}^{\pi} \varphi(r, \theta-t) d \mu(t) \rightarrow D \mu(\theta) \text { as } r \rightarrow 1-
$$

PROOF: The usual approximate identity proof with the Poisson kernel $P_{r}(\theta-t)$ (see for example [1] page 4) works for $p(r, \theta-t)$ as well.

Without loss of generality we may assume that $\theta=0$. Let $A=D \mu(0)$ and

$$
u\left(r e^{i \theta}\right)=\frac{1}{2 \pi} \int_{-\pi}^{\pi} p(r, \theta-t) d \mu(t) .
$$

then

$$
\begin{aligned}
u\left(r e^{i \theta}\right)-A & =\frac{1}{2 \pi} \int_{-\pi}^{\pi} p(r, t)[d \mu(t)-A d t] \\
& =\frac{1}{2 \pi}\left[p(r, t)[\mu(t)-A t]_{-\pi}^{\pi}\right. \\
& -\frac{1}{2 \pi} \int_{-\pi}^{\pi}(\mu(t)-A t) \frac{\partial p}{\partial t} d t
\end{aligned}
$$


where $\mu(t)$ is the function of Bounded Variation associated with $\mu$. Note that the first term tends to 0 as $r \rightarrow 1-$. Fix $\delta>0$ and let $\delta \leq|t| \leq \pi$. Then

$$
\left|\frac{\partial \varphi}{\partial t}(r, t)\right| \leq \frac{\left(1-r^{2}\right)^{3}}{16 r^{2}} \frac{1}{\sin ^{6} \delta / 2}
$$

Hence for each $\delta>0$,

$$
u\left(r e^{i \theta}\right)-A-I_{\delta} \rightarrow 0
$$

as $\mathbf{r} \rightarrow 1-$, where

$$
I_{\delta}=-\frac{1}{2 \pi} \int_{-\delta}^{\delta}(\mu(t)-A t) \frac{\partial \varphi}{\partial t} d t=\frac{1}{\pi} \int_{0}^{\delta}\left[\frac{\mu(t)-\mu(-t)}{2 t}-A\right] t\left(\frac{-\partial \varphi}{\partial t}\right) d t
$$

Given $\epsilon>0$, chose $\delta>0$ such that

$$
\left|\frac{\mu(t)-\mu(-t)}{2 t}-A\right|<\frac{\epsilon}{2} \text { for } 0<t \leq \delta .
$$

Then $\left|I_{\delta}\right| \leq \frac{\epsilon}{2 \pi} \int_{0}^{\pi} t\left|-\frac{\partial \varphi}{\partial t}\right| d t$.

But then $\frac{\partial}{\partial t} p(r, t)=-\frac{\left(1-r^{2}\right)^{3}}{\left(1+r^{2}\right)} \frac{4 r \sin t}{\left(1-2 r \cos t+r^{2}\right)^{3}}$.

Hence $\left|I_{\delta}\right| \leq \frac{\epsilon}{2 \pi} \int_{0}^{\pi} t\left(-\frac{\partial \varphi}{\partial t}(r, t)\right) d t$

$$
\begin{aligned}
& =-\frac{\epsilon}{2 \pi}[t p(r, t)]_{0}^{\pi}+\frac{\epsilon}{2 \pi} \int_{0}^{\pi} p(r, t) d t \\
& \leq \frac{\epsilon}{2 \pi} \int_{-\pi}^{\pi} p(r, t) d t=\epsilon .
\end{aligned}
$$

Now we are ready to prove the main result of this paper. We may recall that if $f$ $\epsilon \mathrm{H}^{\infty}$ then the radial limits, $\lim f\left(\mathrm{re}^{\mathrm{i} \theta}\right)=\mathrm{f}\left(\mathrm{e}^{\mathrm{i} \theta}\right)$ exists for almost all $\theta$ and that

$$
f\left(r e^{i \theta}\right)=\frac{1}{2 \pi} \int_{-\pi}^{\pi} \frac{\left(1-r^{2}\right)}{1-2 r \cos (\theta-t)+r^{2}} f\left(e^{i t}\right) d t
$$


Taking derivatives with respect to $r$, we get

$$
e^{i \theta} f^{\prime}\left(r e^{i \theta}\right)=I_{1}\left(r e^{i \theta}\right)-I_{2}\left(r e^{i \theta}\right),
$$

where $I_{1}\left(r e^{i \theta}\right)=\frac{1}{2 \pi} \int_{-\pi}^{\pi} \frac{-2 r}{1-2 r \cos (\theta-t)+r^{2}} f\left(e^{i t}\right) d t$ and $I_{2}\left(r e^{i \theta}\right)=\frac{1}{\pi} \int_{-\pi}^{\pi} \frac{\left(1-r^{2}\right)(r-\cos (\theta-t))}{\left(1-2 r \cos (\theta-t)+r^{2}\right)^{2}} f\left(e^{i t}\right) d t$. Note that $(1-r) I_{1}\left(r e^{i \theta}\right) \rightarrow$ $-f\left(\mathrm{e}^{\mathrm{i} \theta}\right)$ for almost all $\theta$ as $\mathrm{r} \rightarrow 1-$. Also

$$
\begin{aligned}
I_{2}(1-r) & =\frac{1}{\pi}(1-r) \int_{-\pi}^{\pi} \frac{\left(1-r^{2}\right)(-\cos (\theta-t)+1)}{\left(1-2 r \cos (\theta-t)+r^{2}\right)^{2}} f\left(e^{i t}\right) d t \\
& -\frac{(1-r)^{2}}{\pi} \int_{-\pi}^{\pi} \frac{\left(1-r^{2}\right)}{\left(1-2 r \cos (\theta-t)+r^{2}\right)^{2}} f\left(e^{i t}\right) d t
\end{aligned}
$$

The first term in $I_{2}(1-r)$ is dominated by $(1-r)\|f\|_{\infty}$ and hence tends to zero as $r \rightarrow 1-$. However the second term of $I_{2}(1-r)$ is

$$
=-\frac{2\left(1+r^{2}\right)}{(1+r)^{2}} \frac{1}{2 \pi} \int_{-\pi}^{\pi} p(r, \theta-t) f\left(e^{i t}\right) d t \rightarrow-f\left(e^{i \theta}\right)
$$

for almost all $\theta$ as $\mathrm{r} \rightarrow 1$ - by Lemma 2 .

The Proof is complete.

\section{A BLASCHKE PRODUCT NOT IN $s_{0}$.}

In this section, for completeness sake we give an explicit example of an bounded analytic function which is not in ${ }_{0}{ }_{0}$.

First we state an elementary lemma, whose proof we omit.

LEMMA 3. Let $0<r<1$. Then

(a) If $0<x<r<1$, then $-\ln (1-x)<\frac{x}{1-r}$

(b) $\frac{1-x}{1-x r}$ decreases on $-\infty<x<\frac{1}{r}$

(c) $\frac{1+x}{1-x r}$ increases on $-\infty<x<\frac{1}{r}$.

LEMMA 4. Let $a_{n}=1-\frac{1}{2^{n}}$ and $2 \dot{p}_{n}=a_{n}+a_{n+1}$ with $n \geq 1$. 
Then $\varliminf_{m \rightarrow \infty} \prod_{n=1}^{\infty}\left|\frac{b_{m}-a_{n}}{1-\bar{a}_{n} b_{m}}\right| \geq c>0$ for some $c$.

PROOF: Fix $m \geq 1$. We first show that

$$
\sum_{m+1}^{\infty}-\ln \left|\left[\frac{a_{n}-\beta_{m}}{1-\overline{a_{n}} \beta_{m}}\right]\right| \leq 32
$$

For,

$$
\begin{aligned}
& \sum_{m+1}^{\infty}-\ln \left|\left[\frac{a_{n}-\beta_{m}}{1-\bar{a}_{n} \beta_{m}}\right]\right|=\sum_{m+1}^{\infty}-\ln \left(1-\frac{\left(1-a_{n}\right)\left(1+\beta_{m}\right)}{1-a_{n} \beta_{m}}\right) \\
& \quad \leq\left[\frac{1-a_{m+1} \beta_{m}}{a_{m+1}-\beta_{m}}\right] \sum_{m+1}^{\infty} \frac{\left(1-a_{n}\right)\left(1+\beta_{m}\right)}{1-\beta_{m}} \text {. (use Lenma 3 (a) and (b)). }
\end{aligned}
$$

Then

$$
\sum_{m+1}^{\infty}-\ln \left|\left[\frac{a_{n}-\beta_{m}}{1-\bar{a}_{n} \beta_{m}}\right]\right| \leq \frac{\left(1-\left|a_{m}\right|^{2}\right)}{\frac{1}{2}\left(\alpha_{m+1}-a_{m}\right)} \frac{2}{\left(1-\beta_{m}\right)} \sum_{m+1}^{\infty} \frac{1}{2^{n}} \leq 32 .
$$

Now applying Lemma $3(\mathrm{c})$ to $\frac{1+\mathrm{x}}{1-\mathrm{x} \beta_{\mathrm{m}}}$, we have $\frac{\left(1-\beta_{\mathrm{m}}\right)\left(1+\alpha_{\mathrm{n}}\right)}{1-a_{\mathrm{n}} \beta_{\mathrm{m}}} \leq \frac{\left(1-\beta_{\mathrm{m}}\right)\left(1+\alpha_{\mathrm{m}}\right)}{1-a_{\mathrm{m}} \beta_{\mathrm{m}}}$ for $1 \leq n \leq m$.

Thus,

$$
\begin{aligned}
& \sum_{n=1}^{m}-\ln \left|\frac{\left(a_{n}-\beta_{m}\right.}{1-a_{n} \beta_{m}}\right|=\sum_{n=1}^{m}-\ln \left[1-\frac{\left(1-\beta_{m}\right)\left(1+a_{n}\right)}{1-a_{n} \beta_{m}}\right] \\
& \leq\left[\frac{1-a_{m}^{\beta}}{\beta_{m}-a_{m}}\right] \sum_{n=1}^{m} \frac{\left(1-\beta_{m}\right)\left(1+a_{n}\right)}{1-a_{n} \beta_{m}} \text { by Lerma } 3(a),(c) .
\end{aligned}
$$

Hence;

$$
\begin{aligned}
\sum_{n=1}^{m}-\ln \left|\left[\frac{a_{n}-\beta_{m}}{1-a_{n} b_{m}}\right]\right| & \leq\left[\frac{1-a_{m m}^{\beta}}{\beta_{m}-a_{m}}\right]\left(1-\beta_{m}\right) \sum_{n=1}^{m} \frac{2}{1-a_{n}} \\
& \leq \frac{1-a_{m}^{2}}{\frac{1}{2}\left(\alpha_{m+1}-a_{m}\right)}\left(1-\beta_{m}\right) \sum_{1}^{m} 2^{n+1} \leq 64 .
\end{aligned}
$$


Now Lemma 3 follows.

COROLLARY 1. Let $b$ be the Blaschke product with zeros $\left\{a_{n}: n \geq 1\right\}$. Then

$$
b^{\prime}(r)(1-r) 0 \text { as } \quad r \rightarrow 1-\text {. }
$$

PROOF: Let $c>\prod_{r \rightarrow 1-}\left|b^{\prime}(r)\right|(1-r)$.

Then for sufficiently large $n$,

$$
\left|\mathrm{b}\left(\beta_{\mathrm{n}}\right)\right| \leq \mathrm{c} \int_{a_{\mathrm{n}}}^{\beta_{\mathrm{n}}} \frac{1}{1-\mathrm{r}} \mathrm{dr} \leq \mathrm{c} \int_{a_{\mathrm{n}}}^{a} \mathrm{n}+1 \frac{1}{1-\mathrm{r}} \mathrm{dr}=\mathrm{c} \ln 2 .
$$

Hence by Lemma 4 ,

$$
\varlimsup_{r \rightarrow 1-}\left|b^{\prime}(r)\right|(1-r) \neq 0
$$

REFERENCES

1. DUREN, Peter L., Theory of $\mathrm{H}^{\mathrm{p}}$ spaces, Academic Press, 1970.

2. Garnett, John B., Bounded Analytic Functions, Academic Press, 1981. 


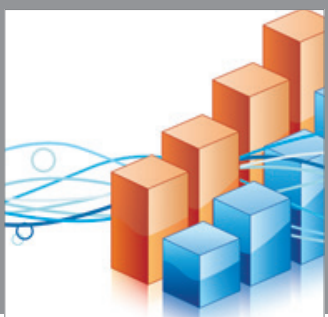

Advances in

Operations Research

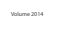

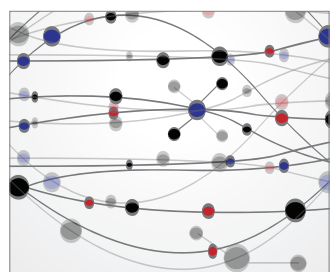

\section{The Scientific} World Journal
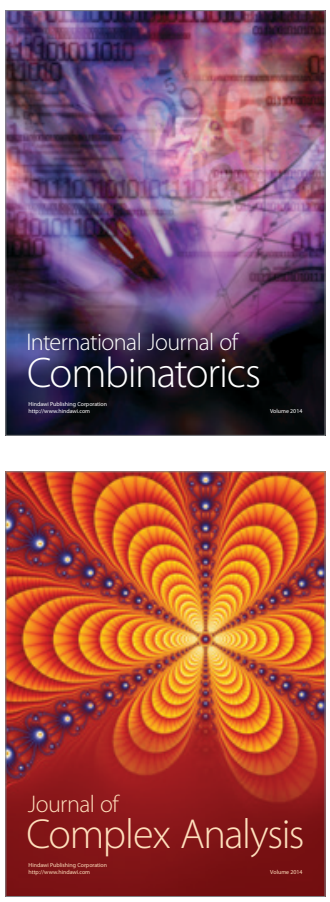

International Journal of

Mathematics and

Mathematical

Sciences
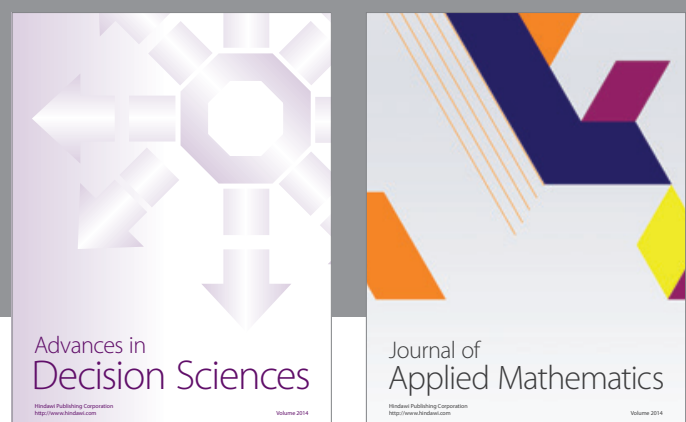

Journal of

Applied Mathematics
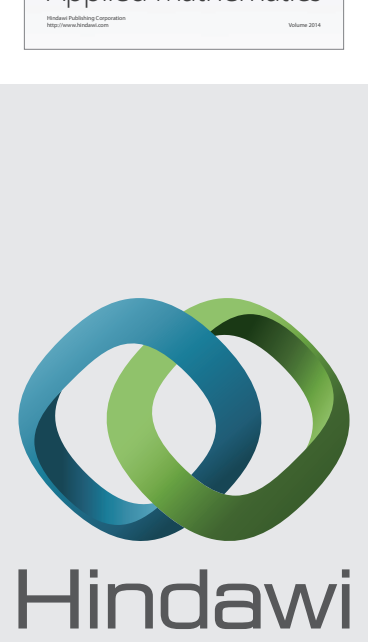

Submit your manuscripts at http://www.hindawi.com
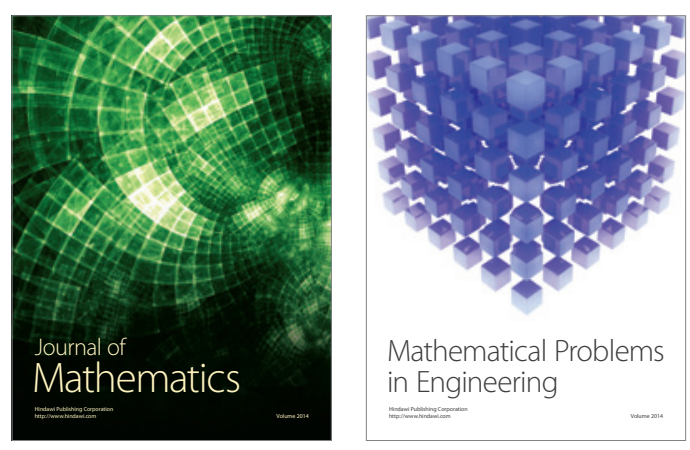

Mathematical Problems in Engineering
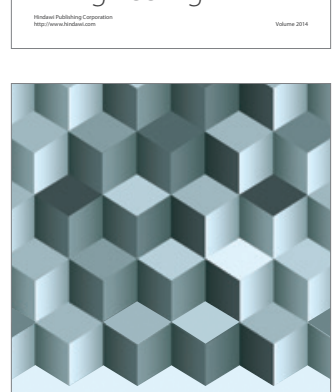

Journal of

Function Spaces
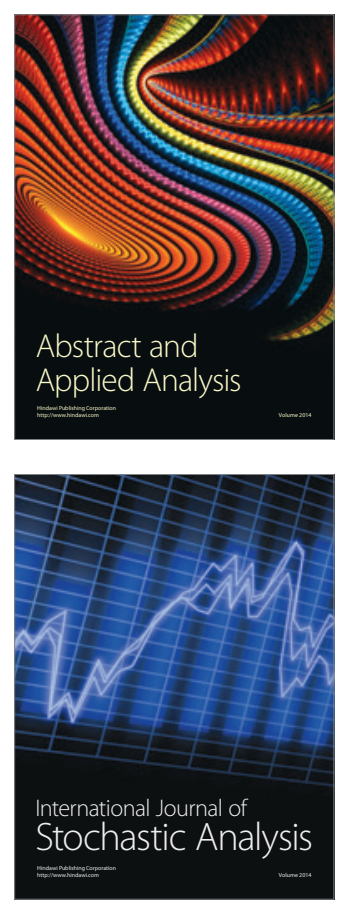

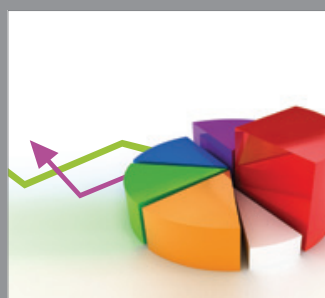

ournal of

Probability and Statistics

Promensencen
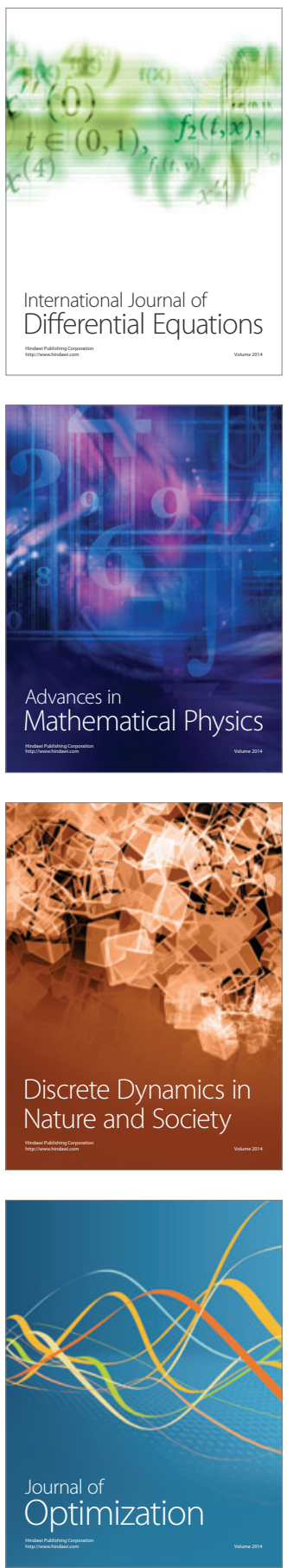